\title{
Research on Fabrication of Nasal Prosthesis based on Computer Vision
}

\author{
Jin Sun ${ }^{\mathrm{a},}$, Yu Ding ${ }^{\mathrm{a}}$, Li Zeng ${ }^{\mathrm{a}}$,Guangxiang Sun ${ }^{\mathrm{b}}$,Zhida Zhu ${ }^{\mathrm{a}}$, Lifeng Zhang ${ }^{\mathrm{c}}$ \\ ${ }^{a}$ College of Mechanical Engineering, Yangzhou University, 196\#,West Huayang Road,Hanjiang District, Yangzhou , \\ Jiangsu, 225127 ,China \\ ${ }^{\mathrm{b}}$ Daqiao Hospital , 1\#,Dongda Road, Jiangdu District, Yangzhou , Jiangsu, 225127 ,China \\ ${ }^{\mathrm{c}}$ Department of Electrical Engineering and Electronics ,Kyushu Institute of Technology , Sensui-cho 1-1, Tobata-ku, \\ Kitakyushu-shi, Fukuoka, 225127 , Japan \\ *Corresponding Author: sunjin1001@126.com
}

\begin{abstract}
In this paper, a novel method for designing and manufacturing of nosal prosthesis is presented. Using the method, multiple point sets of the patient's and volunteer's faces can be generated by 3D white light inspection system based on special binocular cameras without any damages on their skin. Based on CAD technique, volunteer's nose mesh was registered and fit in the patient's face mesh .After be merged, the undersurface of the nasal prosthesis mesh can match the remnant tissue of deformed side perfectly. By rapid prototyping manufacturing machine, the nasal prosthesis can be produced with resin and in good agreement with the deformity area. In so far, integrating non-contact white light inspection,reverse engineering with rapid prototyping manufacturing technology and without any damages on patient's skin, this approach allows us to generate custom-made nasal prostheses easily and rapidly .
\end{abstract}

Keywords: nasal prosthesis, fabrication, computer vision.

\section{Introduction}

There are many reasons for facial defects, mainly cancer, trauma and congenital factors. The facial defects can be classified as the type with symmetrical and asymmetrical feature. The type with asymmetrical feature including: nasal defect, lip defect. Although tissue engineering technology has painted a fascinating prospect for the organization of the organs defect, there is quite a long way to be applied to clinic. Therefore, many facial defect had to use artificial prosthesis. With the development of a variety of materials of prostheses, magnetic attachment retention and implant technology, that facial prostheses need to meet with the requirement of shape, color, texture, function and repair simulation reconstruction facial prostheses has become a main trend. For example, orbital defect, the key is not only to restore the eyelid surface morphology, but also accurately determine location of the eyeball.

With the development of computer technology, computer-aided design and manufacturing, reverse engineering, rapid prototyping and some other new technologies were used in the field of facial prostheses. This paper will provide an integrated technology on how to use non-contact 3D white light inspection, reverse engineering and rapid prototyping technology to design and manufacture nasal prosthesis.

\section{Methods}

\subsection{Workflow}

Different from traditional technologies, this technology generates multiple point sets of patient's and volunteer's faces by 3D white light inspection system based on special binocular Cameras without any damages on their skin . Based on CAD technique, volunteer's nose mesh was registered and fit in the patient's face mesh .After be merged, the undersurface of the nasal prosthesis mesh can match the remnant tissue of deformed side perfectly. By rapid prototyping manufacturing machine, the nasal prosthesis can be produced with resin and in good agreement with the deformity area. The basic workflow see Fig 1. At present, prosthesis was fabricated for a patient who had nasal defect and left craniofacial defect. The fabrication of nasal prosthesis applying this technology can be divided into four steps: 
1.Data acquisition of patient's and volunteer's face;

2. Data preprocessing;

3. Prosthesis slip creating;

4. Prosthesis making.

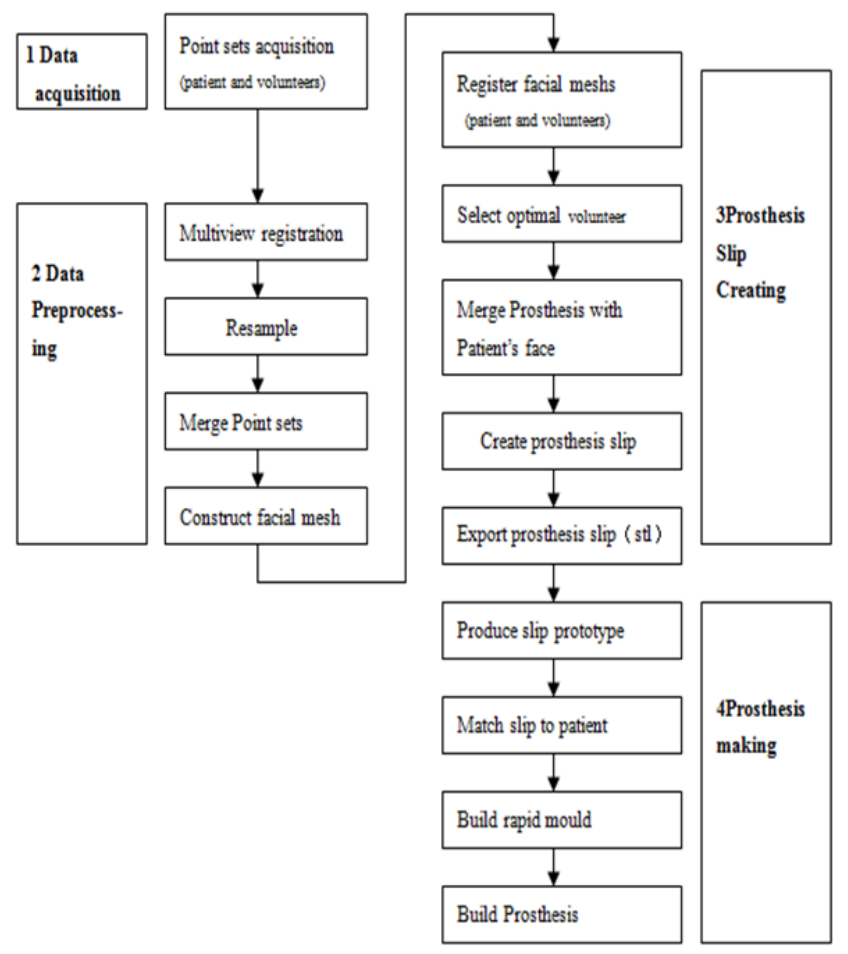

Fig.1. Workflow of fabrication of nasal prosthesis.

\subsection{Data acquisition}

Here is 3D white light inspection system, see Fig.2. With three-dimensional structure of optical projection vision measurement technology, this system can be used in collecting nearly 2 million points data of patients' facial soft tissue under non-contact and natural state conditions within 10 seconds; Its measuring accuracy reaches less than $0.03 \mathrm{~mm}$;Measured by special binocular Cameras, a facial measurements can be obtained with complete data, avoiding to produce multi-angle data pieced error; By physical coding grating projection, you will get high-contrast, a row intensity distribution of the projection stripes .In addition, because of white light source, there is no harm to human body.

This system can provide practical and reliable service in the measurement of human's face, which owns an important clinical value. As shown in Fig. 3 is the appearance of measurement system measurement system. and Fig.4 is measurement and control system flow chart.

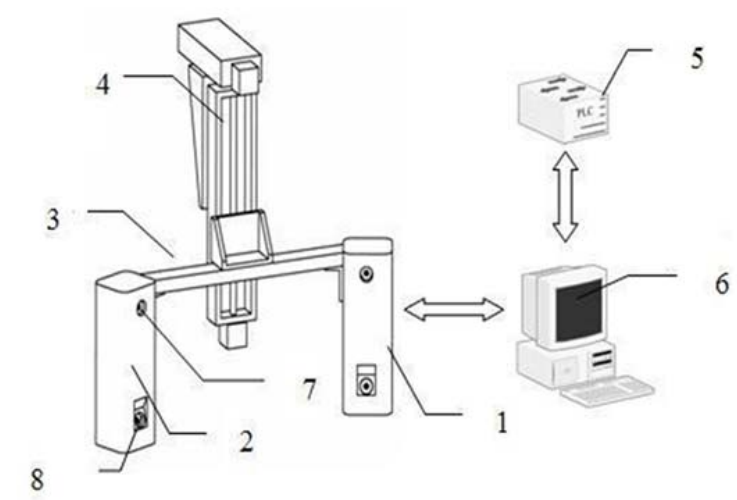

Fig. 2. Overview of measurement system. 1 Left CCD; 2 Right CCD; 3 Bracket ;4 Slide; 5 PLC; 6 Console; 7 Camera; 8 Projector.

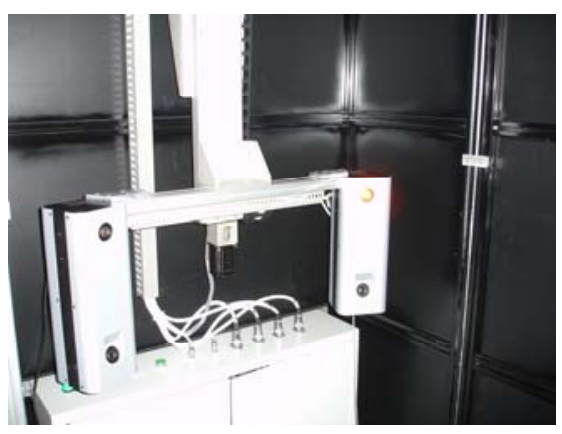

Fig. 3. Appearance of measurement system.

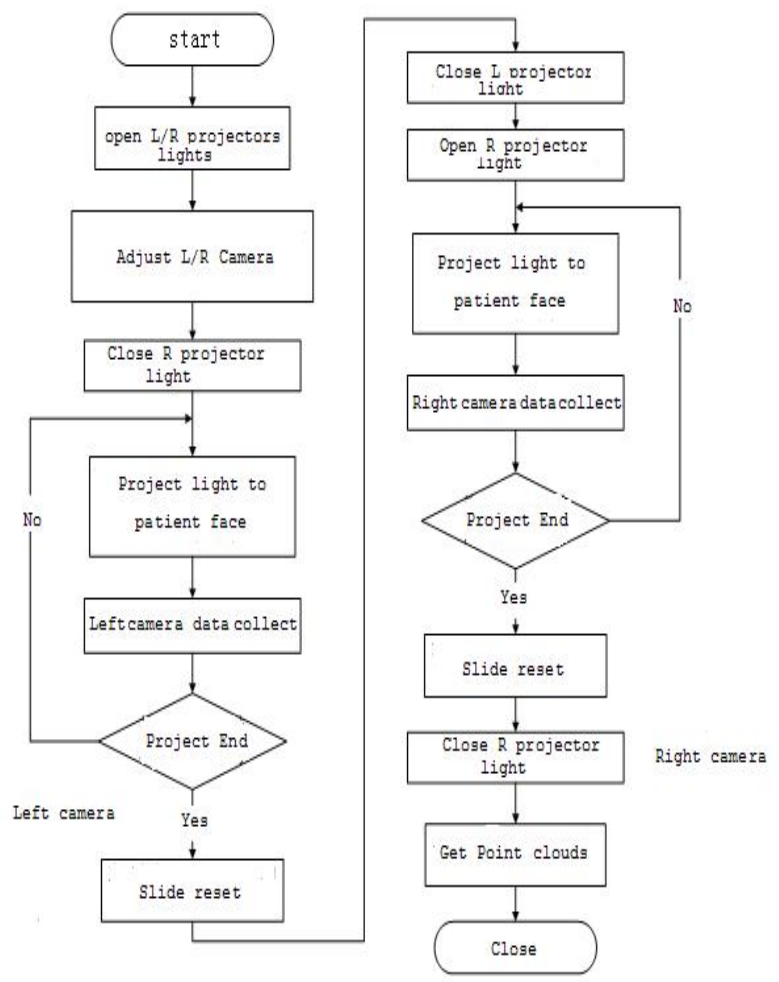

Fig. 4. The flowchart of the measurement process. 


\subsection{Data preprocessing}

Point sets processing included removal of unwanted point, multi-view registration, re-sampling, coordinates and normalization, and so on. After meeting with the required accuracy by coarse registration and global registration, point sets were been pieced together, see Fig.5, then been built into facial mesh.

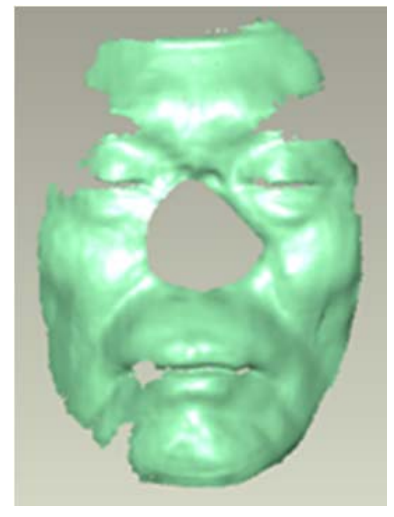

(a) Patient's.

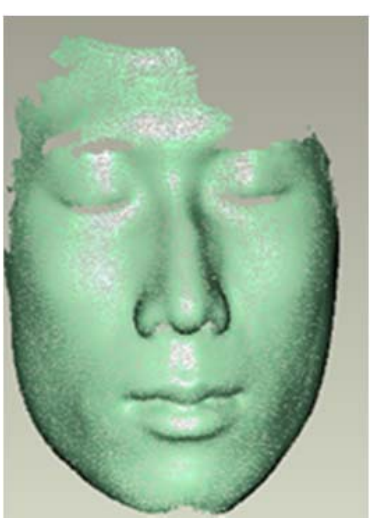

(b) Volunteer's.
Fig. 5. Facial meshes after data preprocessing.

\subsection{Prosthesis slip Creating}

After acquiring initial facial mesh of patient and volunteers, used ICP arithmetic to register facial meshs between patient and volunteers, as shown in Fig .6.

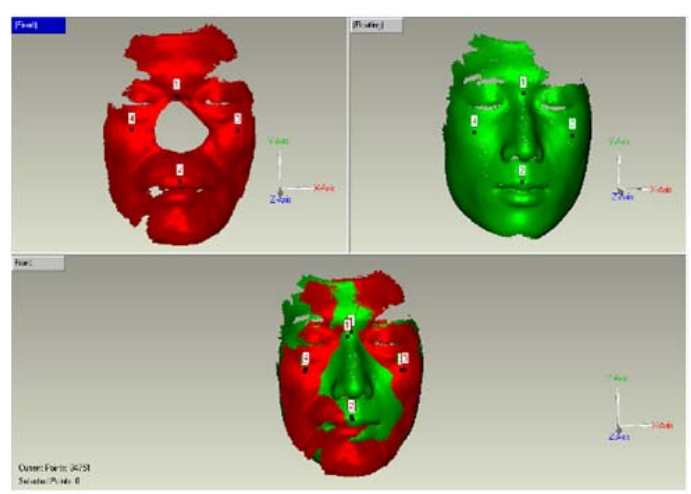

Fig. 6. Register facial meshs (patient's and volunteer's)

Through comparing gaps between facial meshs between patient and volunteers in forehead/ cheeks, the volunteer who had smallest comprehensive gap from patient was selected as the optimal facial mesh.After merge prosthesis with patient's face, extruded prosthesis slip with $2 \mathrm{~mm}$ thickness then exported them by format of stl.

\subsection{Prosthesis slip Creating}

Used rapid prototyping mechanism to build Prosthesis slip, then matched Prosthesis slip to patient's facial surfaces and repaired, see Fig. 7(a) . Utilized wax to fix Prosthesis slip to create a rapid mould of Prosthesis; After opening the rapid mould,allocated medical silicone in accordance with patient's skin color and when their color was very close, you can build nasal prosthesis in a vacuum machine-up system. Fig.7(b) is the effect when the patient wore the nasal prosthesis.

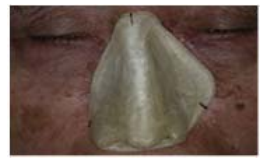

(a) nasal prosthesis

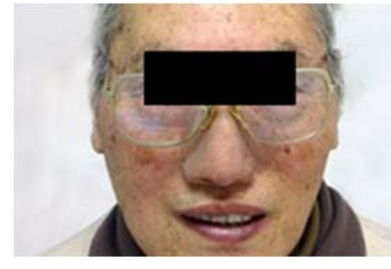

(b) final result
Fig. 7. The nasal prosthesis and final result

\section{Discussion}

In traditional system of hand-carved stage, based on wax, there were a range of issues such as: production of complex, long production cycle, poor aesthetic effects, so production of prostheses to a large extent depend on individual technicians’ ability of artfacial .

In last two decades, many scholars tried various methods in producing facial prostheses ${ }^{(1-2)}$, for example, Cheah ${ }^{(3)}$ used CAD / CAM technology in treatment of facial defect. He first received 3D shape facial defect data by using laser scanners, then got the CAD model of the defect through data process and geometric reconstruction, and at last he built the resin prototype by using lithography methods; Mitsuhiro Tsuji ${ }^{(4)}$ produced eye prosthesis in 2002; G. Dimitroulis ${ }^{(5)}$ made nasal prosthesis in 2007; Using similar approach, X. Huang. $\mathrm{M}^{(6)}$ cured auricular defects by CT image, but there were still some deficiencies, such as:

1) Laser scanning technology Its flaw is : slow to meet with facial expression change and potential harm to the human skin.

2) CT 、 MRI Its disadvantage is low spatial resolution, poor measurement accuracy, slow access to data ,high costs for clinical use and particularly defects on soft tissue because of their scan of radiation;

In order to overcome above deficiencies, we have proposed a framework to design and manufacturing of nasal prosthesis, there are some merits: the white light has no harm to human body; patient's face can be measured within 10 seconds and nearly 2 million points data can be gotten; measuring accuracy reaches less than $0.03 \mathrm{~mm}$. 


\section{Copyright}

Papers submitted to ICIAE must describe original and unpublished researches. They should not have been submitted to other conferences or journals. Any paper which is under review elsewhere will not be considered for publication. Once an eligible paper is accepted, it will be published under a Creative Commons Attribution 3.0 (CC BY 3.0) License.

\section{Conclusions}

Integrating non-contact white light inspection ,reverse engineering with rapid prototyping manufacturing technology , a new project of nasal prosthesis making was proposed : using 3D white light inspection system, multiple point sets of the patient's and volunteer's faces can be generated by based without any damages on their skin. Based on CAD technique, selected volunteer's nose mesh was registered and fit in the patient's face mesh.After be merged, the undersurface of the nasal prosthesis mesh can match the remnant tissue of deformed side perfectly. This technique can be expected to flourish to meet the highest demands of comfort, precision, and safety.

\section{Acknowledgment}

This work was supported by National Natural Science Foundation of China(Grant No. 51475409), Research Project of State Key Laboratory of Mechanical System and Vibration(Grant No. MSV201810), Yangzhou city Yangzhou University of Science and Technology Cooperation Program Funds(Grant No. YZ2016244), 2016 Six talent peaks project in Jiangsu Province (Grant No. JXQC-030).

\section{References}

(1) K. F. Leong, C. M. Cheah, and C. K. Chua: "Solid freeform fabrication of three-dimensional prostheses for engineering replacement tissues and organs”, Biomaterials, vol.24, pp. 2363-2378, 2003

(2) C. M. Cheah, C. K. Chua, K. H. Tan, and C. K. Teo: "Integration of laser surface digitising with computer-aided design and manufacturing for developing facial prostheses-Part 2: Development of moulding techniques for casting prosthetic parts", International Journal of Prosthodontics, Vol.16, No.5, pp.541-546, 2003

(3) C. M. Cheah, C. K. Chua, and K. H. Tan: "Integration of laser surface digitizing with CAD/CAM techniques for developing facial prostheses. part 1: design and fabrication of prostheses replicas”, The International Journal of Prosthodontics, vol.16, No.5, pp.435-441, 2003

(4) M. Tsuji, N. Noguchi, K. Ihara, Y. Yamashita, M. Shikimori, and M. Goto: "Clinical reports. fabrication of a maxillofacial prosthesis using a computer-aided design and manufacturing system", Journal of Prosthodontics, vol. 13, pp. 179-183, 2004.

(5) G. Dimitroulis: "Nasal implants following nasectomy", International Journal of Oral \& Maxillofacial Surgery, vol. 36, pp. 447-449, 2007.

(6) X. Huang, M. Ye, W. Zhang, C. Wang, and T. Jiao: "Fabricating auricular prostheses based on rapid prototyping and the FreeForm modelling system" , The International Journal of Advanced Manufacturing Technology, vol. 24, pp. 873-878, 2004. 